\title{
Outcomes of a Physician-Controlled Wire-Guided Cannulation of the Bile Duct Using a Novel Sphincterotome: A Single-Center, Prospective Study
}

\author{
Koichiro Mandaia, b, Koji Uno ${ }^{a}$, Yuki Ueda ${ }^{\text {a }}$, Yusuke Okada ${ }^{a}$, Kenjiro Yasuda ${ }^{a}$
}

\begin{abstract}
Background: Efficacy of a physician-controlled wire-guided cannulation of the bile duct has been reported. This study aimed to evaluate a novel sphincterotome with a short tip and a short wire, which can be bowed compactly compared to conventional sphincterotomes.
\end{abstract}

Methods: This was a single-arm, single-center, prospective study. We enrolled patients with choledocholithiasis and/or obstructive jaundice and/or cholangitis for whom endoscopic sphincterotomy was planned. The main outcome measurement was the proportion of successful selective bile duct cannulation by physician-controlled wireguided cannulation within $10 \mathrm{~min}$.

Results: The study cohort consisted of 40 patients. The proportion of successful selective bile duct cannulation within 10 min was $72.5 \%$ $(n=29)$. The proportion of post-endoscopic retrograde cholangiopancreatography pancreatitis was $2.5 \%($ mild, $n=1)$ and the proportion of hemorrhage was $2.5 \%$ (moderate, $\mathrm{n}=1$ ).

Conclusions: Although physician-controlled wire-guided cannulation using a novel sphincterotome with a short tip and a short wire was not superior to wire-guided cannulation using conventional sphincterotomes reported in a previous study, it remains a safe and efficacious alternative in terms of successful biliary cannulation.

Keywords: Endoscopic retrograde cholangiopancreatography; Novel sphincterotome; Physician-controlled wire-guided cannulation; Selective bile duct cannulation

\section{Introduction}

Selective bile duct cannulation (SBDC) is an essential tech-

Manuscript submitted January 25, 2018, accepted February 8, 2018

a Department of Gastroenterology, Kyoto Second Red Cross Hospital, 355-5 Haruobi-cho, Kamigyo-ku, Kyoto 602-8026, Japan

${ }^{\mathrm{b} C}$ Corresponding Author: Koichiro Mandai, Department of Gastroenterology, Kyoto Second Red Cross Hospital, 355-5 Haruobi-cho, Kamigyo-ku, Kyoto 602-8026, Japan. Email: mndkchr@gmail.com

doi: https://doi.org/10.14740/gr974w nique for endoscopic retrograde cholangiopancreatography (ERCP)-related procedures. The injection method using a conventional ERCP catheter loaded with contrast medium has been the standard method for SBDC for a long time. Recently, the usefulness of the wire-guided cannulation (WGC) technique has been reported [1-3].

Several randomized, controlled trials (RCT) have shown that WGC facilitated selective bile duct cannulation and decreased the incidence of post-ERCP pancreatitis (PEP) [1,2]. The recent Japanese RCT showed that WGC led to significantly shorter SBDC and fluoroscopic time during the procedure, although WGC did not improve the proportion of successful SBDC and did not reduce the occurrence of PEP [3]. The recent RCT demonstrated that physician-controlled wire-guided cannulation (PCWGC) was associated with a lower incidence of endoscopic complications, such as PEP, than assistant-controlled wire-guided cannulation [4]. Hence, PCWGC should be considered the first-line method for managing bile duct cannulation. Especially in patients for whom endoscopic sphincterotomy (EST) is planned, the WGC technique with a sphincterotome is efficient because it leads to quick guidewire insertion and EST, and because the sphincterotome bow is available to obtain the correct axis for bile duct cannulation.

Recently, a novel sphincterotome with a short tip and a short wire, called the CleverCut3V Short Bend Tip, was introduced to easily perform PCWGC. The CleverCut3V Short Bend Tip, the tip of which can be bowed compactly compared with conventional sphincterotomes, has been designed to provide a closer approach to the papilla and allow for cannulation while in a suboptimal scope position.

The present study aimed to evaluate the efficacy and safety of PCWGC with this novel sphincterotome in patients for whom EST is planned.

\section{Materials and Methods}

\section{Study design}

This was a single-arm, single-center, prospective study registered as an interventional study with the University hospital Medical Information Network (UMIN) (registration number: UMIN 000024153). Patients were recruited from October 2016 to April 2017. The study protocol was reviewed and ap- 


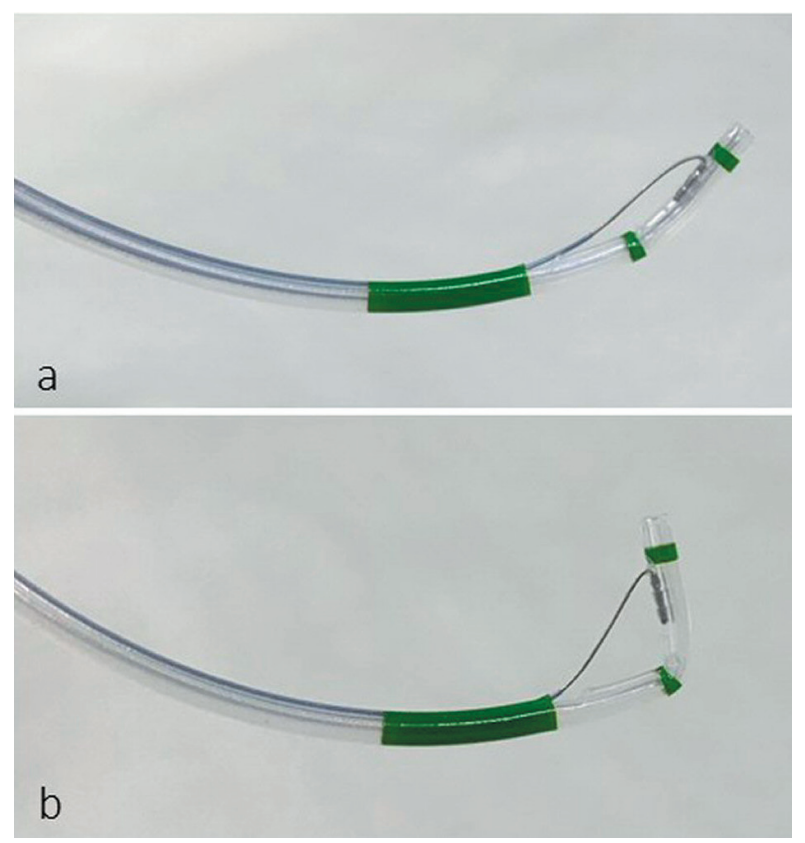

Figure 1. (a) CleverCut3V Short Bend Tip with a tip length of $2 \mathrm{~mm}$ and a cutting wire length of $15 \mathrm{~mm}$. (b) The tip can be bowed compactly.

proved by the Kyoto Second Red Cross Hospital Institutional Review Boards and conformed to the tenets of the Declaration of Helsinki. All participants gave written informed consent.

\section{Patients}

Inclusion criteria for selecting patients were as follows: 1) patients aged 20 years or older, 2) patients with choledocholithiasis and/or obstructive jaundice and/or cholangitis, 3) patients in whom ERCP and EST were planned, and 4) patients who had native papilla. Obstructive jaundice was defined as either a total bilirubin level $\geq 2.0 \mathrm{mg} / \mathrm{dL}$ or high levels of enzymes of the hepatobiliary system (alkaline phosphatase (ALP), $\gamma$-glutamyltransferase $(\gamma$-GT), aspartate aminotransferase (AST), and alanine aminotransferase (ALT) levels $>1.5$ times the upper limit of normal value) observed with a blood test and obstruction to bile flow by the stones or tumors diagnosed by an imaging study. Cholangitis was defined as either fever $\left(>38^{\circ} \mathrm{C}\right)$ or inflammation (white blood cell count $>10,000 /$ $\mu \mathrm{L}$ or C-reactive protein level $\geq 1 \mathrm{mg} / \mathrm{dL}$ ) with either a total bilirubin level $\geq 2.0 \mathrm{mg} / \mathrm{dL}$ or elevated hepatobiliary enzyme levels (ALP, $\gamma$-GT, AST, and ALT $>1.5$ times the upper limit of normal value). A papilla was considered native if EST or endoscopic papillary dilation and/or stent placement was not previously performed.

Patients were excluded from this study if they met any of the following criteria: 1) patients who had an ampullary tumor, 2) patients who had tumor invasion in the papilla, 3) patients with surgically altered gastrointestinal or pancreatobiliary anatomy, 4) patients with a value $>3$ in performance status, according to the scale of the Eastern Cooperative Oncology Group [5], 5) patients with severe systematic disease graded as higher than American Society of Anesthesiologists physical status III, 6) dialyzed patients, and 7) patients judged to have $<$ 3 months of life expectancy.

\section{Study protocol}

ERCP was performed using a 15-degree backward oblique angle duodenoscope (BOAD) with an elevator function (JF260V; Olympus Medical Systems Corp., Tokyo, Japan). A CleverCut3V Short Bend Tip (KD-VC412Q-0215, Olympus Medical Systems Corp., Tokyo, Japan) with a tip length of 2 $\mathrm{mm}$ and a cutting wire length of $15 \mathrm{~mm}$ was used as a sphincterotome (Fig. 1). A 0.025-inch guidewire with a straight tip or an angled tip (VisiGlide2, Olympus Medical Systems Corp., Tokyo, Japan) was used as a guidewire.

All operators were endoscopists with ERCP experience of 200 procedures or more and with $\geq 6$ years of experience.

\section{Bile duct cannulation protocol}

All procedures were performed with the patients placed in the prone position and under procedural sedation with midazolam and pethidine. Additionally, propofol was administered to patients who showed a poor response to sedation. After viewing the papilla, bile duct cannulation was initiated using a sphincterotome preloaded with a guidewire in all patients. A guidewire was controlled by an operator, and the use of a contrast medium was not allowed until the sphincterotome was inserted into the bile duct. The guidewire was gently advanced under fluoroscopic guidance. When the guidewire could not be inserted into the bile duct, it was retracted and another attempt was made. When the guidewire was advanced to the bile duct, the tip of the sphincterotome was inserted into the bile duct and SBDC was successfully confirmed after injecting a contrast medium. If SBDC was not achieved within $10 \mathrm{~min}$, the method was changed, such as an injection method using a conventional ERCP catheter or a sphincterotome, or followed the pancreatic guidewire placement method or pre-cut technique.

In all patients, a blood test was performed $2 \mathrm{~h}$ after the procedure and the next morning of the procedure, in order to evaluate the potential complications of the procedure.

\section{Outcome measurements}

The primary endpoint was the proportion of SBDC success by PCWGC within $10 \mathrm{~min}$. Cannulation time was measured from the time of advancement of the sphincterotome from the endoscope channel placed in front of the papilla to the time of the confirmation of SBDC success by a contrast medium injection. The secondary endpoints were as follows: 1) SBDC time, 2) proportion of final successful SBDC, 3) final SBDC technique, 4) complications, and 5) comparison between the SBDC with PCWGC success group and the SBDC with PCWGC failed group. The diagnostic criteria for post-ERCP pancreatitis (PEP) were continuous abdominal pain that lasted for $>24 \mathrm{~h}$ 
Table 1. Patients' Clinical Characteristics

\begin{tabular}{ll}
\hline Median age (SD), years & $72.2(11.4)$ \\
Sex (male), n (\%) & $20(50.0)$ \\
Reasons for ERCP, n & Choledocholithiasis, 29 \\
& Malignant biliary stricture, 10 (pancreatic cancer, 5; bile duct cancer, 2; gallbladder cancer, 2; metastatic lymph nodes, 1) \\
& Benign biliary stricture, 1 \\
\hline
\end{tabular}

SD: standard deviation.

after the procedure and hyperamylasemia 3 times greater than the upper limit of normal. Severity was judged in accordance with Cotton's criteria [6].

The analyzed population excluded patients in whom the papilla could not be reached.

\section{Sample size}

The previous Japanese RCT showed that the proportion of successful SBDC within 10 min was $71.3 \%$ by the injection method with an ERCP catheter, $73.5 \%$ by the WGC technique with an ERCP catheter, $68 \%$ by the injection method with a sphincterotome, and $69.1 \%$ by the WGC technique with a sphincterotome [3]. Hence, the threshold proportion of successful SBDC within 10 min was set at $60 \%$ and the expected proportion at $80 \%$. Using Simon's optimal two-stage design, we estimated that 13 patients were to be assessed first, and if more than eight patients were successful, then 22 additional patients were to be added to establish a significance level of $5 \%$ and statistical power of $80 \%$. We assumed that about $10 \%$ of enrolled patients would drop out of this study; the target number of enrolled patients was therefore set at 40 .

\section{Statistical analysis}

We used Fisher's exact tests to compare the proportions of categorical variables (such as sex) between the groups. The threshold for significance was $\mathrm{P}<0.05$. All statistical analyses were performed using SPSS version 22 (IBM Corp., Armonk, NY).

\section{Results}

Thirteen patients were initially enrolled, of which SBDC was successful in 11, within $10 \mathrm{~min}$. Thereafter, we enrolled 27 more patients to obtain a cohort of 40 patients. No patients dropped out of this study.

\section{Patients}

Patients' characteristics are presented in Table 1 . The analyzed population consisted of 20 men with a mean age of 72.2 years (range, 42 - 93 years). The reasons for ERCP were choledocholithiasis $(n=29)$, malignant biliary stricture $(n=10)$, and benign biliary stricture $(\mathrm{n}=1)$.

\section{SBDC outcomes}

The proportion of SBDC success within 10 min by PCWGC with a CleverCut3V Short Bend Tip was $72.5 \%(n=29)$ (Table 2). The median time for SBDC was $240 \mathrm{~s}$ (Table 2). SBDC was successful in $97.5 \%(\mathrm{n}=39)$ of patients. The final SBDC techniques used in 10 patients in whom SBDC success required $>$ 10 min were contrast injection with an ERCP catheter $(n=5)$, contrast injection with a CleverCut3V Short Bend Tip $(n=1)$, pancreatic guidewire placement $(\mathrm{n}=1)$, and precutting $(\mathrm{n}=$ 3) (Fig. 2). The following complications were observed in the analyzed population: mild PEP in $2.5 \%(\mathrm{n}=1)$ and moderate hemorrhage in $2.5 \%(\mathrm{n}=1)$ (Table 2).

\section{Factors affecting SBDC failure by PCWGC with a Clever- Cut3V Short Bend Tip}

To investigate factors affecting SBDC failure by PCWGC with a CleverCut3V Short Bend Tip, we compared the SBDC with PCWGC success group to the SBDC with PCWGC failed group. The definition of a large oral protrusion was as follows: an oral protrusion with three or more folds, or a marked swelling oral protrusion (hooknose shape), which had an indistinct border between the papilla and the oral protrusion (Fig. 3) [7]. As shown in Table 3, patients who had a papilla with a large oral protrusion were significantly greater in the failed group than in the success group (odds ratio, 16.000; 95\% confidence interval, 1.537 - 166.533; $\mathrm{P}=0.015)$.

\section{Discussion}

This study investigated the proportion of SBDC success within 10 min by PCWGC with a CleverCut3V Short Bend Tip in

Table 2. SBDC Success Within $10 \mathrm{~min}$, SBDC Time, and Complications

\begin{tabular}{ll}
\hline SBDC success within 10 min, n (\%) & $29(72.5)$ \\
Median SBDC time, s (IQR) & $240(92-925)$ \\
Complications, n (\%) & Pancreatitis mild, 1 (2.5) \\
& Hemorrhage moderate, $1(2.5)$ \\
\hline
\end{tabular}

SBDC: selective bile duct cannulation; IQR: interquartile range. 


\section{Clinical course}

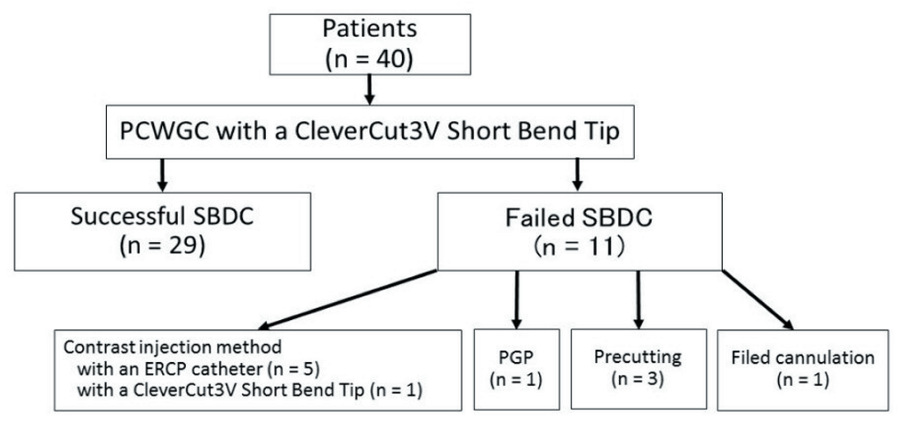

PCWGC, physician-controlled wire-guided cannulation; SBDC, selective bile duct cannulation; ERCP, endoscopic retrograde cholangiopancreatography; PGP, pancreatic guidewire placement method

Figure 2. The proportion of final SBDC success was $97.5 \%(n=39)$. The final SBDC techniques used in 10 patients with failed PCWGC were contrast injection method $(n=6)$, PGP $(n=1)$, and precutting $(n=3)$.
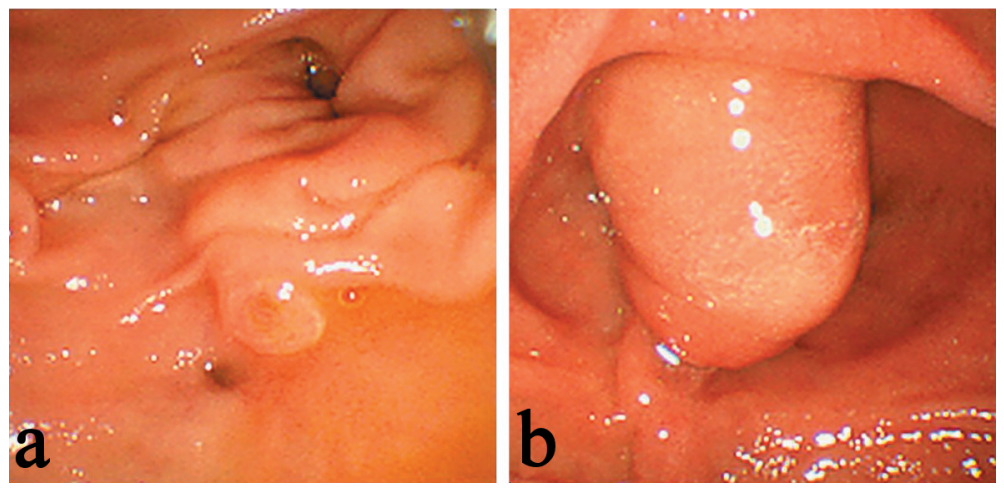

Figure 3. (a) An oral protrusion with three or more folds. (b) A marked swelling oral protrusion (hooknose shape).

patients for whom EST was planned. The CleverCut3V Short Bend Tip has been designed to provide a closer approach to the papilla and allow for cannulation while in a sub-optimal scope position, and it has a tip length of $2 \mathrm{~mm}$ and a cutting wire length of $15 \mathrm{~mm}$. We found that the proportion of SBDC success within 10 min was similar to that reported in a previous RCT [3]. There were no severe complications.

Previous studies have reported that the proportion of SBDC success within 10 min by WGC with a sphincterotome was $69.1-77.9 \%$ [3, 8]. The proportion of SBDC success within 10 min of our study was not superior to that of the previous studies. Although the tip of the CleverCut3V Short Bend Tip can be bowed compactly, compared with conventional sphinc- terotomes, this advantage did not bring about an improvement in the proportion of SBDC success. This was an unexpected result but may be plausible because the previous RCT reported that bile duct cannulation was favorable without bowing the papillotome tip in using a 15 -degree BOAD, whereas adjusting the angle by bowing the papillotome tip improved the proportion of SBDC success when using a 5-degree BOAD [9]. Further investigation in patients for whom a 5-degree BOAD is used is needed.

According to previous studies, the reasons for SBDC difficulty were reported to be a juxtapapillary diverticulum [10], a malignant biliary stricture due to pancreatic cancer [11], and a papilla with a large oral protrusion [12]. In this study, pa-

Table 3. Factors Affecting SBDC Failure by PCWGC With a CleverCut3V Short Bend Tip

\begin{tabular}{llllll} 
& $\begin{array}{l}\text { SBDC success with } \\
\text { PCWGC (n= 29) }\end{array}$ & $\begin{array}{l}\text { SBDC failure with } \\
\text { PCWGC (n= 11) }\end{array}$ & $\begin{array}{l}\text { Odds } \\
\text { ratio }\end{array}$ & $\begin{array}{l}\text { Two-tailed } \\
\text { P value }\end{array}$ \\
\hline Malignant biliary stricture due to pancreatic cancer, n $\mathrm{n}$ & 2 & 3 & 5.063 & $0.716-35.776$ & $0.117^{*}$ \\
Juxtapapillary diverticulum, $\mathrm{n}$ & 11 & 5 & 1.364 & $0.335-5.552$ & $0.728^{*}$ \\
Large oral protrusion, $\mathrm{n}$ & 1 & 4 & 16.000 & $1.537-166.533$ & $0.015^{*}$ \\
\hline
\end{tabular}

SBDC: selective bile duct cannulation; PCWGC: physician-controlled wire-guided cannulation; Cl: confidence interval. *Fisher's exact test. 
tients who had a papilla with a large oral protrusion were significantly greater in the failed group than in the success group, demonstrating that PCWGC was not effective in patients who had a papilla with a large oral protrusion. In these patients, guidewire tips may have been stuck in epithelial folds or flexion of intra-duodenal biliary segments [13], and aligning the axis of the cannula with the axis of the lower bile duct assisted by contrast injection or straightening the narrow distal segment by the pancreatic guidewire placement method may be useful.

The limited sample size without a control group and performance of the study in a single center are limitations of this study.

In conclusion, PCWGC with the CleverCut3V Short Bend Tip was not superior to the contrast injection method and WGC reported in a previous RCT in terms of successful biliary cannulation, but it is a safe and efficacious alternative.

\section{Acknowledgments}

The authors thank Kasumi Sanada, Azumi Suzuki, Hironori Wada, Atsushi Shirakawa, Naokuni Sakiyama, and Takuji Kawamura who have contributed to data collection.

\section{Conflict of Interest}

All the authors declare no conflict of interest.

\section{References}

1. Lella F, Bagnolo F, Colombo E, Bonassi U. A simple way of avoiding post-ERCP pancreatitis. Gastrointest Endosc. 2004;59(7):830-834.

2. Artifon EL, Sakai P, Cunha JE, Halwan B, Ishioka S, Kumar A. Guidewire cannulation reduces risk of postERCP pancreatitis and facilitates bile duct cannulation. Am J Gastroenterol. 2007;102(10):2147-2153.

3. Kawakami H, Maguchi H, Mukai T, Hayashi T, Sasaki T, Isayama H, Nakai Y, et al. A multicenter, prospective, randomized study of selective bile duct cannulation performed by multiple endoscopists: the BIDMEN study. Gastrointest Endosc. 2012;75(2):362-372, 372 e361.
4. Buxbaum J, Leonor P, Tung J, Lane C, Sahakian A, Laine L. Randomized trial of endoscopist-controlled vs. assistant-controlled wire-guided cannulation of the bile duct. Am J Gastroenterol. 2016;111(12):1841-1847.

5. Oken MM, Creech RH, Tormey DC, Horton J, Davis TE, McFadden ET, Carbone PP. Toxicity and response criteria of the Eastern Cooperative Oncology Group. Am J Clin Oncol. 1982;5(6):649-655.

6. Cotton PB, Lehman G, Vennes J, Geenen JE, Russell RC, Meyers WC, Liguory C, et al. Endoscopic sphincterotomy complications and their management: an attempt at consensus. Gastrointest Endosc. 1991;37(3):383-393.

7. Horiuchi A, Nakayama Y, Kajiyama M, Tanaka N. Effect of precut sphincterotomy on biliary cannulation based on the characteristics of the major duodenal papilla. Clin Gastroenterol Hepatol. 2007;5(9):1113-1118.

8. Nambu T, Ukita T, Shigoka H, Omuta S, Maetani I. Wire-guided selective cannulation of the bile duct with a sphincterotome: a prospective randomized comparative study with the standard method. Scand J Gastroenterol. 2011;46(1):109-115.

9. Kawakami H, Maguchi H, Hayashi T, Yanagawa N, Chiba A, Hisai H, Amizuka H. A prospective randomized controlled multicenter trial of duodenoscopes with 5 degrees and 15 degrees backward-oblique angle using wireguided cannulation: effects on selective cannulation of the common bile duct in endoscopic retrograde cholangiopancreatography. J Gastroenterol. 2009;44(11):11401146.

10. Zoepf T, Zoepf DS, Arnold JC, Benz C, Riemann JF. The relationship between juxtapapillary duodenal diverticula and disorders of the biliopancreatic system: analysis of 350 patients. Gastrointest Endosc. 2001;54(1):56-61.

11. Mandai K, Uno K, Fujii Y, Kawamura T, Yasuda K. Number of endoscopic retrograde cholangiopancreatography procedures required for short biliary cannulation time. Gastroenterol Res Pract. 2017;2017:1515260.

12. Ban T, Nojiri Y, Fujita Y, Hayashi K, Orito E. Evaluation of the algorithm for selective bile duct cannulation for patient with naive choledocholithiasis. Journal of Japan Biliary Association. 2015;29:57-63 (in Japanese).

13. Omuta S, Maetani I, Shigoka H, Gon K, Saito M, Tokuhisa J, Naruki M. Newly designed J-shaped tip guidewire: a preliminary feasibility study in wire-guided cannulation. World J Gastroenterol. 2013;19(28):4531-4536. 